TITLE:

\title{
INDEX THEOREMS AND MICROSUPPORT
}

$\operatorname{AUTHOR}(S)$ :

Boutet de Monvel, L.

CITATION:

Boutet de Monvel, L.. INDEX THEOREMS AND MICROSUPPORT. 数理解 析研究所講究録 1993, 854: 1-19

ISSUE DATE:

1993-11

URL:

http://hdl.handle.net/2433/83752

RIGHT: 


\section{INDEX THEOREMS AND MICROSUPPORT \\ L. Boutet de Monvel (Univ. Paris VI) 10y 6 大淁}

In these notes we propose to describe again the Atiyah-Singer index theorem for systems of differential operators, and related extensions such as the index theorem for Toeplitz operators or the relative index theorem proved by $\mathrm{B}$. Malgrange and the author. We make a special emphasis on the microlocal contribution produced by the sheaf in which the solutions of the differential equations are computed, as described by P.Schapira and J.P.Schneiders, believing that this point of view, although as yet incomplete, sheds a new and unifying light.

We describe this presentation of the index theorem in $\$ 1$. It was not possible in these short notes to give complete and detailed proofs, and we have limited ourselves to give in 3 appendixes short, but hopefully useful, descriptions of the main ingredients: K-theory, $\mathscr{D}$ modules, and the idea of the proof.

\section{Description of the index theorem}

Let us recall that complex of vector spaces is a sequence

$$
\mathrm{p}: \quad \ldots \rightarrow \mathrm{E}_{\mathrm{k}} \stackrel{\mathrm{p}_{\mathrm{k}}}{\rightarrow} \mathrm{E}_{\mathrm{k}+1} \rightarrow \ldots \quad(\mathrm{k} \in \mathbf{Z})
$$

of complex vector spaces $E_{k}$ and linear maps $p_{k} \in L\left(E_{k} ; E_{k+1}\right)$ such that $p_{k+1}$ o $p_{k}=0$ (we may identify a linear map $p: E_{0} \rightarrow E_{1}$ to a complex of length 2, concentrated in degrees 0 and 1). The cohomology $H^{*}(p)$ is the graded vector space $H^{*}(p)=\operatorname{ker} p / \operatorname{Imp}\left(H^{k}(p)=k e r p_{k} / \operatorname{Im} p_{k-1}\right)$. a is a Fredholm complex if $\mathrm{H}^{*}(\mathrm{p})$ is finite dimensional, ie. the $\mathrm{H}^{\mathrm{k}}(\mathrm{p})$ are finite dimensional and vanish except for a finite number of indices $\mathrm{k}$. Then the index (Euler characteristic) of a is defined as the alternating sum:

$$
\text { Ind } p=\sum(-1)^{k} \operatorname{dim} H^{k}(p)
$$

The index theorem is concerned with the index of complexes in which the $\mathrm{p}_{\mathrm{k}}$ are differential operators on a manifold $X$ and the $E_{k}$ are suitable spaces of distributions on $X$ or parts of $X$. It has long been known that under suitable ellipticity conditions the index exists, and that it is quite stable under small perturbations or deformations, so one expects that it can be computed in terms of simpler topological invariants of the data. We first recall what these formulas look like.

The model for all index formulas is Hirzebruch's formulation of the Riemann-Roch theorem for coherent sheaves on complex projective spaces. In this formula the ingredients 
are the Chern character of the sheaf and the Todd class of the space. Later A.Grothendieck gave a relative version of the Riemann-Roch theorem, in which the topological ingredient is the behaviour under direct image of a $\mathrm{K}$-theoretical element associated to the sheaf. Let us recall the formulation of Baum, Fulton and Mac Pherson of Grothendieck's theorem: let X be a projective analytic space and $Z \subset X$ a subspace. The Grothendieck group $K_{Z}^{a n}(X)$ is the group generated by isomorphy classes of coherent $\mathcal{O}_{\mathrm{X}}$-modules with support in $\mathrm{Z}$, and the relations $[M]=\left[M^{\prime}\right]+\left[M^{\prime \prime}\right]$ for each exact sequence $0 \rightarrow M^{\prime} \rightarrow M \rightarrow M^{\prime \prime} \rightarrow 0$. There is a canonical homomorphism $\mathrm{K}_{Z}^{\mathrm{an}}(\mathrm{X}) \rightarrow \mathrm{K}_{Z}^{\mathrm{top}}(\mathrm{X})$, where $\mathrm{K}_{\mathrm{Z}}^{\mathrm{top}}(\mathrm{X})$ is the Atiyah group of "virtual vector bundles with support in $Z$ ", which describes the additive and deformation invariant properties of complexes of vector bundles which are exact outside of $\mathrm{Z}$. According to Baum, Fulton, Mac Pherson, the relative Riemann-Roch theorem states that this homomorphism commutes to proper direct images (it also commutes to inverse images). This should be complemented by the description of the $\mathrm{K}$-theoretical image, which is constructed by means of the Bott periodicity theorem. One may further translate this in terms of cohomology, using the Chern character:

$$
\mathrm{ch}: \mathrm{K}_{\mathrm{Z}}^{\mathrm{top}}(\mathrm{X}) \otimes \mathbb{Q} \underset{\rightarrow}{\rightarrow} \mathrm{H}_{\mathrm{Z}}^{\mathrm{pair}}(\mathrm{X}, \mathbb{Q})
$$

The Hirzebruch-Riemann-Roch theorem is the cohomological translation when then goal manifold is a point (recall that $\mathrm{E} \rightarrow \operatorname{dim} \mathrm{E}$ defines the canonical isomorphism $\mathrm{K}$ (point) $\simeq \mathbb{Z}$ ). In this analysis the Todd class appears when one interprets the K-theoretical direct image (whose cohomological interpretation is not integration along fibers).

The Atiyah-Singer index formula deals with elliptic complexes of differential operators on a real manifold. Since holomorphic functions are solutions of the elliptic system of Cauchy-Riemann equations, this formula contains the complex Riemann-Roch formula from which it was inspired. Although the first proof of this formula was cohomological and close to that of Hirzebruch, the published proof of 1968 is more inspired by that of Grothendieck and convincingly shows that K-theory is a natural tool in this context.

A similar case where one has an index theorem is the following: if $\mathrm{X}$ is a complex manifold, $U \subset X$ a relatively compact open subset with smooth boundary $\partial U$, and $p$ a complex of holomorphic differential operators on $\mathrm{X}$, one defines the non characteristicity of $\partial \mathrm{U}$ for $\mathrm{p}$; this condition is closely related to the ellipticity condition in the real case, and when it is true, the complex $p$ acting on germs of holomorphic sections near $U \cup \partial U$ is Fredholm and its index is essentially given by the same Riemann-Roch formula (the same formula holds more generally for Toeplitz operators - cf. Boutet de Monvel 1.) 
In these notes we restrict ourselves to operators with analytic coefficients on analytic manifolds. This is not an important resriction for the topological aspect of the index formula because this deals with homotopy classes of continuous functions, which usually contain real-analytic functions. It does make a difference for the analysis and geometry of the differential operators involved : some pathologies are avoided, but mostly in the analytic setting we dispose of a good algebraic and geometric formalism similar to those of algebraic or analytic geometry, which would not exist otherwise, eg. there is a good notion of supports and characteristic sets, and direct or inverse images.

In this setting the data for the index theorem is the following: first we have a complex manifold X ; a real manifold will always be considered as the germ of a subset $X_{0} \subset X$ (the set of real points in a complex manifold). On $\mathrm{X}$ we have a differential system with analytic coefficients, best described as a coherent $\mathscr{D}$-module $\mathcal{M}(\mathscr{D}$ denotes the sheaf of analytic differential operators). Finally we have a sheaf of coefficients $\mathscr{F}$ in which we compute the solutions. We will represent the differential system by a sheaf $\mathscr{M}$ of right $\mathscr{D}$-modules (or more generally by an object with coherent cohomology of the derived category of these); $\mathscr{F}$ is a sheaf of left $\mathscr{D}$-modules (or more generally an object of the derived category); the sheaf of solutions is the complete tensor product $\mathcal{M}_{\otimes_{\mathscr{D}}}^{\llcorner} \mathscr{F}$ (an object of the derived category of sheaves of vector spaces), and the index (if it exists) is the alternating sum of the Betti numbers of its global sections:

(4) $\operatorname{Index}(\mathscr{M}, \mathscr{F})=\chi\left(\mathscr{M}^{\mathrm{L}} \stackrel{\otimes}{\mathscr{D}}^{\mathcal{F}}\right)=\Sigma(-1)^{\mathrm{i}} \operatorname{dim} \mathrm{R}^{\mathrm{i}} \Gamma\left(\mathrm{X}, \mathcal{M}_{\otimes_{\mathscr{D}}^{\mathrm{L}}}^{\mathscr{F}}\right)$

(One can equivalently represent the differential system by a left $\mathscr{D}$-module, the sheaf of solutions being $\operatorname{RHom}(\mathcal{M}, \mathscr{F}))$.

Let us point out typical cases for the sheaf $\mathscr{F}$ of coefficients: a first example is the case where $\mathscr{F}$ is the sheaf $\mathcal{O}$ of holomorphic functions; in this case (X complex compact), we may take $\mathscr{M}=m \otimes_{\mathcal{O}} \mathscr{D}$ with $m$ a coherent $\mathscr{D}$-module, and the index theorem will give back the Riemann-Roch theorem (in fact one gets a little more since coherent left $\mathscr{D}$-modules are not all of the form $m \otimes_{\mathscr{O}} \mathscr{D}$ ). More generally one can choose $\mathscr{F}$ a coherent left $\mathscr{D}$-module; the ellipticity condition is then that SS $\mathcal{M}$ and SSF meet along a compact set (in the zero section of $\left.T^{*} X\right)$. The index formula is then the formula below.

A second example is the following: let $\mathrm{Y}$ be a closed subset of $\mathrm{X}$ and $\mathscr{F}=\mathcal{O}_{\mathrm{Y}}$ the sheaf of germs of holomorphic functions along $\mathrm{Y}\left(\mathscr{F}=\mathrm{i}_{*} \mathrm{i}^{-1} \mathcal{O}\right.$ if $\mathrm{i}$ is the canonical inclusion $\mathrm{Y} \rightarrow \mathrm{X}$, so that for the stalks we have $\mathscr{F}_{\mathrm{x}}=\mathcal{O}_{\mathrm{x}}$ if $\mathrm{x} \in \mathrm{Y}$, and 0 otherwise). The case of Toeplitz operators corresponds to the case where $\mathrm{Y}=\mathrm{U} \cup \partial \mathrm{U}$ with $\mathrm{U}$ a relatively compact open subset 
of $X$, with smooth boundary $\partial \mathrm{U}$. The case of operators on a real manifold corresponds to the case where $\mathrm{Y}=\mathrm{X}_{0}$ is the set of real points of $\mathrm{X}$ as above. More generally Schapira and Schneiders have examined the case $\mathscr{F}=\mathscr{O} \otimes f$ where $f$ is a real-constructible sheaf.

We may now describe the structure of the index formula: to $\mathcal{M}$ is associated its microsupport SS $\mathcal{M}$, and a $\mathrm{K}$-theoretical element $[\mathscr{M}] \in \mathrm{K}_{\mathrm{SS} \mathscr{M}}\left(\mathrm{T}^{*} \mathrm{X}\right)$ (when $\mathscr{M}$ is given as a complex of differential operators with symbol exact outside of $Z=S S \mathscr{M},[\mathscr{M}]$ is the element of $\mathrm{K}_{\mathrm{SS} \mathscr{M}}\left(\mathrm{T}^{*} \mathrm{X}\right)$ defined by the symbol ; in general cf. Boutet de Monvel-Malgrange). In the examples described above we can also define the microsupport SSF , and in the best cases a $\mathrm{K}$-theoretical element $[\mathscr{F}] \in \mathrm{K}_{\mathrm{SS} \mathscr{F}}\left(\mathrm{T}^{*} \mathrm{X}\right)$. The ellipticity condition is that $\mathrm{SS} \mathcal{M} \cap S S \mathscr{F}$ is compact. Then the index formula then reads as follows:

Index theorem : $\quad$ (5) $\operatorname{Index}(\mathcal{M}, \mathscr{F})=\chi^{\mathrm{top}}([\mathcal{M}][\mathscr{F}])$

where $\chi^{\text {top }}$ is the canonical $\mathrm{K}$-theoretical character $\mathrm{K}_{Z}\left(\mathrm{~T}^{*} \mathrm{X}\right) \rightarrow \mathbf{Z}$ arising from the complex structure of $\mathrm{T}^{*} \mathrm{X}$ (cf. appendix 1 ), and $[\mathcal{M}][\mathscr{F}]$ is the $\mathrm{K}$-theoretical product (it has compact support SS $\mathscr{M} \cap S S \mathscr{F}$ ). In the cases we will describe below $\mathscr{F}$ is associated to a simple set $\mathrm{U}$,

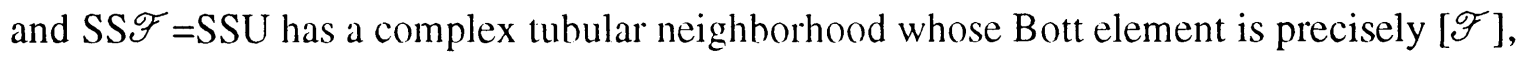
so the index formula can be rewritten

(5)bis $\quad \operatorname{Index}(\mathscr{M}, \mathscr{F})=\chi^{\mathrm{top}}([\mathcal{M}] \mid S S \mathscr{F})$

If $\mathscr{F}$ is $\mathscr{D}$-coherent, $S S \mathscr{F}$ is its charateristic set as mentionned; if $\mathscr{F}=\mathscr{O} \otimes f$ with $f$ a real-constructible sheaf, SSF is the microsupport of $f$ as defined by Kashiwara and Schapira. The index formula in the case $\mathscr{F}=\mathscr{O} \otimes f$ with $f$ a real-constructible sheaf was described by Schapira and Schneiders, in terms of the microlocal Euler classes of $f$ and $\mathscr{M}$. Here we have described the index formula in terms of K-theory; this seems more natural in view of Grothendieck's and Atiyah-Singer's work, and also for formulas with parameters. At this stage this description is not complete, although it contains all previous cases of the index theorem: we need to associate to the sheaf of coefficients $\mathscr{F}$ a K-theoretical element $[\mathscr{F}] \in \mathrm{K}_{\mathrm{SS} \mathscr{F}}\left(\mathrm{T}^{*} \mathrm{X}\right)$. This was done by M.Ohana only in the simpler cases $\mathscr{F}=\mathcal{O}_{\mathrm{Y}}$ if $\mathrm{Y}$ is a real-analytic submanifold with comers of $\mathrm{Y}$; it remains to be done in more general cases.

To conclude let us point out that the preceeding description also applies to the relative case, as described in Boutet de Monvel-Malgrange: let $\mathrm{f}: \mathrm{X} \rightarrow \mathrm{Y}$ be a submersion of complex analytic manifolds. Then one defines the transfer module $\mathscr{D}_{X \rightarrow Y}$ (the sheaf on $\mathrm{X}$ of "differential operators" of type $\mathcal{O}_{\mathrm{Y}} \rightarrow \mathcal{O}_{\mathrm{X}}: \varphi \rightarrow \mathrm{P}\left((\mathrm{Q} \varphi)_{\mathrm{O}}\right.$ f) with $\mathrm{P}$, resp. Q, a differential operator on $\mathrm{X}$, resp. $\mathrm{Y}) . \mathscr{D}_{\mathrm{X} \rightarrow \mathrm{Y}}$ is a coherent $\mathscr{D} \mathrm{X}$-module and its characteristic set is the set 
$\mathrm{H} \simeq \mathrm{T}^{*} \mathrm{Y} \times{ }_{\mathrm{Y}} \mathrm{X}$ of horizontal covectors. Let $\mathrm{U} \subset \mathrm{X}$ be an open set with smooth boundary $\partial \mathrm{U}$ : then $\mathrm{SSO}_{\mathrm{U}}$ was defined above (it is the zero section above $\mathrm{U}$ and the set of outgoing normal

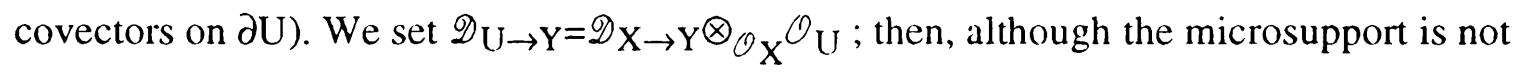
generally defined in this context, a reasonable definition is $S S \mathscr{D}_{\mathrm{U} \rightarrow \mathrm{Y}}=\mathrm{SS} \mathscr{D}_{\mathrm{X} \rightarrow \mathrm{Y}}+\mathrm{SS} \mathscr{O}_{\mathrm{U}}$ (at

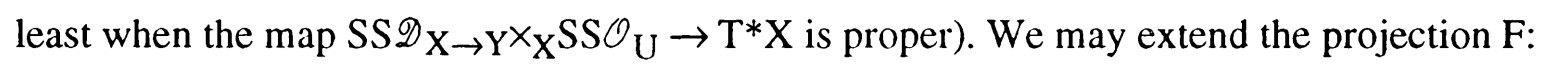
$S S D_{X} \rightarrow Y=T^{*} Y \times_{Y} X \rightarrow T^{*} Y$ to a map $F_{e}: S S D_{U \rightarrow Y}$ by requiring that it is constant along real half-lines parallel to the normal outgoing real half-lines along $\partial U$.

When $\mathscr{M}$ is a coherent $\mathscr{D}$-module on X, we studied in Boutet de Monvel-Malgrange the direct image of the germ of $\mathcal{M}$ along UUวU:

$$
\mathrm{f}_{\mathrm{U}}{ }^{+}(\mathscr{M})=\mathrm{Rf}_{*}\left(\mathscr{M} \otimes_{\mathscr{D}} \mathscr{D}_{\mathrm{U} \rightarrow \mathrm{Y}}\right)
$$

(which describes intuitively the differential relations on Y between germs of solutions of $\mathscr{M}$ along the fibers of $\mathrm{f}$ in $\mathrm{U} \cup \partial \mathrm{U})$. The geometric ellipticity condition is that $S S \mathcal{M} \cap S S \mathscr{D}_{\mathrm{U} \rightarrow \mathrm{Y}}$ is contained in $\mathrm{H}$; the geometric finiteness condition is that the restriction $\mathrm{Fe}$ :

$S S M \cap \cap S S \mathscr{D}_{\mathrm{U} \rightarrow \mathrm{Y}} \rightarrow \mathrm{T}^{*} \mathrm{Y}$ is proper. The relative ellipticity condition (with respect to $\mathrm{U}$ ) is that any section of $\mathscr{M}$ is killed by some vertical operator which is non-characteristic along $\partial U$ and it cannot be read on the principal symbol alone. It implies geometric ellipticity, and together with the stronger compactness condition above, as was shown by Houzel-Schapira, it implies relative finiteness, ie. that $\left.\mathrm{f}_{U^{+}}{ }^{+} \mathscr{M}\right)$ has coherent cohomology. In this case we have $\operatorname{SSf}_{\mathrm{U}}{ }^{+}(\mathcal{M}) \subset \mathrm{Z}=\mathrm{F}_{\mathrm{e}}\left(\mathrm{SS} \mathcal{M} \cap S S \mathscr{D}_{\mathrm{U} \rightarrow \mathrm{Y}}\right)$ and the relative index formula of Boutet de MonvelMalgrange can be written:

\section{Relative index formula: (7) $\quad\left[\mathrm{f}_{\mathrm{U}}^{+}(\mathcal{M})\right]_{\mathrm{Z}}=\mathrm{F}_{\mathrm{e} *}[\mathcal{M}]_{S S \mathscr{M}} \mid \mathrm{SS} \mathscr{D}_{\mathrm{U} \rightarrow \mathrm{Y}}$}

where $\mathrm{F}_{\mathrm{e} *}$ is the $\mathrm{K}$-theoretical image (the relation to the formula above is that the product $[\mathscr{M}]_{S S \mathscr{M}}\left[S S \mathscr{D}_{\mathrm{U} \rightarrow \mathrm{Y}}\right]$ corresponds to $[\mathcal{M}]_{S S \mathcal{M}} \mid \mathrm{SS} \mathscr{D}_{\mathrm{U} \rightarrow \mathrm{Y}}$ by the Bott isomorphism from $\mathrm{K}_{\mathrm{W}}\left(\mathrm{SS} \mathscr{D}_{\mathrm{U} \rightarrow \mathrm{Y}}\right)$ to $\mathrm{K}_{\mathrm{W}}\left(\mathrm{T}^{*} \mathrm{X}\right)$ with $\left.\mathrm{W}=\mathrm{SS} \mathcal{M} \cap S S \mathscr{D}_{\mathrm{U} \rightarrow \mathrm{Y}}\right)$ 


\section{Appendix 1. K theory}

a. Definitions Let $\mathrm{X}$ be a paracompact topological space. We recall that the Atiyah group $\mathrm{K}(\mathrm{X})$ is the group generated by isomorphy classes of vector bundles and the groupoïd law $\oplus$. If $\mathrm{Z} \subset \mathrm{X}$ a closed subset, $\mathrm{K}_{\mathrm{Z}}(\mathrm{X})$ is the group of equivalence classes [a] of bounded complexes a of $\mathbf{C}$-vector bundles on $\mathrm{X}$, exact outside of $\mathrm{Z}$, where the equivalence relation is generated by the relations:

(i) $\quad[\mathrm{a}]+[\mathrm{b}]=[\mathrm{a} \oplus \mathrm{b}]$

(ii) $[\mathrm{a}]=0$ if there exists a deformation of a to an exact complex, exact out of $\mathrm{Z}$.

In fact any element of $\mathrm{K}_{Z}(\mathrm{X})$ is the class of length 2 : if

$$
\mathrm{a}: \quad \ldots \rightarrow \mathrm{E}_{\mathrm{k}} \stackrel{\mathrm{ak}}{\rightarrow} \mathrm{E}_{\mathrm{k}+1} \rightarrow \ldots \quad(\mathrm{k} \in \mathrm{Z})
$$

is a bounded complex on $X$ exact out of $Z$, we have $[a]=\delta(a) \in \mathrm{K}_{Z}(X)$, where $\delta(a)$ is the operator (= complex of length 2$) a+a^{*}: \sum E^{2 k} \rightarrow \sum E^{2 k+1}$.

$\mathrm{K}$-theory is equipped with a product, corresponding to the tensor product of complexes of vector bundles: if $u \in K_{Z}(X)$ and $v \in K_{Z^{\prime}}(X)$ then $u v \in K_{Z \cap Z^{\prime}}(X)$; in particular $K(X)$ in an algebra and $\mathrm{K}_{\mathrm{Z}}(\mathrm{X})$ id a $\mathrm{K}(\mathrm{X})$-module.

Let $\mathrm{H}$ be a Hilbert space and let Fred $(\mathrm{H}) \subset \mathrm{L}(\mathrm{H})$ be the set of Fredholm operators. If $\mathrm{Z} \subset \mathrm{X}$ as above we denote $\mathrm{F}_{\mathrm{Z}}(\mathrm{X})$ the group of homotopy classes of continuous functions $\mathrm{A}$ : $X \rightarrow$ Fred $(H)$ invertible outside of $Z$. Since $G L(H)$ is contractible (by N.Kuiper's theorem) this is identical the group of homotopy classes of Fredholm Hilbert bundles which are exact outside of $\mathrm{Z}$ (a complex of Hilbert bundles is Fredholm if its cohomology is finite dimensional at each point). There is an obvious map $\mathrm{K}_{Z}(X) \in \mathrm{F}_{\mathrm{Z}}(\mathrm{X})$ because a finitedimensional complex is a particular case of Fredholm Hilbert complex. Jänisch's theory shows that this is an isomorphism if $\mathrm{Z}$ is compact, or if the Cech dimension of $\mathrm{X}$ is finite. The inverse map is the index map and is denoted Ind $_{Z}$.

b. Inverse image If $\mathrm{f}$ is a continuous map $\mathrm{X} \rightarrow \mathrm{Y}$, the inverse image for vector bundles

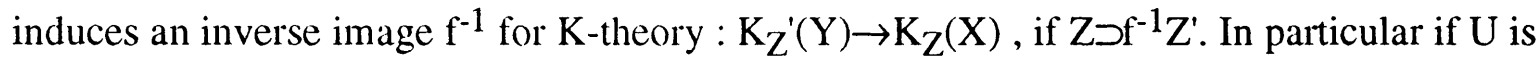
an open set of $X$ and $Z \subset U$ is closed in $X$, there is a restriction map $K_{Z}(X) \rightarrow K_{Z}(U)$. If $X$ is finite dimensional this is always an isomorphism (excision) (a Fredholm map a on $U$ invertible outside of $\mathrm{Z}$ can be deformed into 1 outside of some small neighborhood of $\mathrm{Z}$ by Kuiper's theorem, so its homotopy class can be extended).

c. Products K-theory is multiplicative: if $\xi=[a] \in \mathrm{K}_{Z}(X)$ and $\eta=[b] \in \mathrm{K}_{Z}[X]$ where a , resp. $b$ are two complexes of vector spaces (resp. or Fredholm complexes), exact outside of 
$Z$ resp. $Z^{\prime}$, then $\xi \eta=[a \otimes b] \in K_{Z \cap Z^{\prime}}(X)$, where $a \otimes b$ is the tensor product of complexes (it is exact wherever one of the factors is). Slightly more generally if we have $Z \subset Y \subset X$, and $\xi=[a] \in K_{Z}(Y), \eta=[b] \in K_{Y}(X)$, we define the product $\xi \eta \in K_{Z}(X):$ it is the class $[\tilde{a} \otimes b]$ where $\tilde{a}$ is any extension of a to $X$ (if $a$ is a Fredholm family, we may take any extension, Fredholm or not, of $\mathrm{a}$ : the product $\widetilde{a} \otimes \mathrm{b}$ will still be exact (thus Fredholm) outside of $\mathrm{Y}$ because $b$ is).

d. Bott isomorphism If $\mathrm{N}$ is a complex vector bundle on $\mathrm{X}$, we define its Koszul complex, which is a complex of vector bundles on $\mathrm{N}$ considered as a topological space:

$$
\mathrm{k}_{\mathrm{N}} \quad \ldots \Lambda^{-\mathrm{k}}\left(\mathrm{p}^{-1} \mathrm{~N}^{*}\right) \rightarrow \Lambda^{-\mathrm{k}+1}\left(\mathrm{p}^{-1} \mathrm{~N}^{*}\right) \rightarrow \ldots \rightarrow\left(\mathrm{p}^{-1} \mathrm{~N}^{*}\right) \rightarrow \mathrm{C} \rightarrow 0
$$

where $\mathrm{p} \mathrm{N} \rightarrow \mathrm{X}$ is the projection, $\mathrm{N}^{*}$ the dual bundle of $\mathrm{N}$, and the diferential at a point $n \in N$ is the interior product $\omega \rightarrow \mathrm{n}_{\mathrm{L}} \omega$.

$\mathrm{k}_{\mathrm{N}}$ is exact outside of the zero section (which we identify with $\mathrm{X}$ ) and defines an element $\left[\mathrm{k}_{\mathrm{N}}\right] \in \mathrm{K}_{X}(\mathrm{~N})$. The Bott map $\beta_{\mathrm{NX}}$ is the $\mathrm{K}$-theoretical multiplication by $\left[\mathrm{k}_{\mathrm{N}}\right]$

$$
\beta_{\mathrm{NX}}: \mathrm{K}_{\mathrm{Z}}(\mathrm{X}) \rightarrow \mathrm{K}_{\mathrm{Z}}(\mathrm{N})
$$

Atiyah's formulation of the Bott periodicity theorem is that (if $\mathrm{X}$ is finite dimensional) this map is always an isomorphism (cf Boutet de Monvel-Malgrange for the case where the support $\mathrm{Z}$ is $\neq \mathrm{X}$ ). The proof in Boutet de Monvel-Malgrange describes the inverse map as the index of families of Toeplitz operators. Thus the Bott periodicity theorem appears as the first and fundamental case of all index theorems, and essentially all proofs of the index theorem consist in reducing to this formula.

e. K-theoretical image We end this section by the description of the K-theoretical push-forward. Let us notice that the Bott element $\left[\mathrm{k}_{\mathrm{N}}\right]$ is as well defined by the bundle map

$$
\delta\left(\mathrm{k}_{\mathrm{N}}\right)=\mathrm{k}_{\mathrm{N}}+\mathrm{k}_{\mathrm{N}}^{*}: \beta_{\mathrm{N}}^{+} \rightarrow \beta_{\mathrm{N}}
$$

where $\beta_{\mathrm{N}}^{+}=\Lambda^{\text {even }} \mathrm{N}^{*}$ and $\beta_{\mathrm{N}}^{-}=\Lambda^{\text {odd }} \mathrm{N}^{*}$ (we have chosen some hermitian metric to define the adjoint $\mathrm{k}_{\mathrm{N}}^{*}$ ). Thus the Bott element and the Bott map are still well defined if $\mathrm{N}$ is only a real vector bundle equipped with a spin ${ }^{c}$ structure. ${ }^{l} \operatorname{Spin}^{c}$ structures also give rise to a

$\hat{\mathrm{I}}^{1} \mathrm{~A} \operatorname{spin}^{\mathrm{c}}$ structure on a real vector bundle $\mathrm{N}$ consists of a euclidean metric on $\mathrm{N}$, and a simple graded Clifford bundle (ie a vector bundle $s_{N}=s_{N}^{+}+s_{N}^{-}$endowed with a structure of $C_{N}$-module, where $C_{N}$ is the Clifford algebra of $N$ - generated by $N$ and the relations $n . n=-\|n\|^{2}$ for each $n \in N$, which is simple at each point of $\mathrm{X}$ ) 


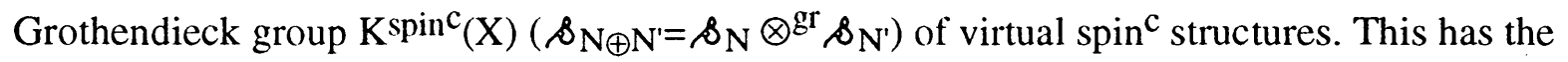
following property (which does not hold for complex structures): if $\mathrm{E}$ is a real vector bundle, and $\xi \in \mathrm{K}^{\operatorname{spin}^{\mathrm{c}}}(\mathrm{X})$ a virtual $\operatorname{spin}^{\mathrm{c}}$ structure with underlying real virtual bundle [E], then there is a unique compatible $\operatorname{spin}^{\mathrm{c}}$ structure on $\mathrm{E}^{2}$

If $\mathrm{X}$ and $\mathrm{Y}$ are two smooth manifolds and $\mathrm{f}$ a differentiable map $\mathrm{X} \rightarrow \mathrm{Y}$, a spinc structure on $f$ is a spin ${ }^{c}$ structure on the normal bundle $N(f)=f^{-1}[T Y]-[T X] \in K(X)$. A map between complex (or symplectic) manifolds carries a canonical $\operatorname{spin}^{\mathrm{c}}$ structure.

For $f$ spin $^{c}$ and proper on $Z \subset X$, the K-theoretical push-forward $K_{Z}(X) \rightarrow K_{f(Z)}(Y)$ is defined by the following axioms:

- it is covariant: $(\mathrm{fg})_{*}=\mathrm{f}_{*} \mathrm{~g}_{*}$

- if $\mathrm{f}: \mathrm{X} \rightarrow \mathrm{N}$ is the zero section of a spinc bundle (equipped with the obvious spinc structure), then $\mathrm{f}_{*}$ is the Bott isomorphism.

- it is compatible with a change of basis, ie. if we have a diagram

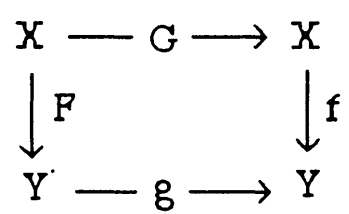

where $X, Y, X^{\prime}, Y^{\prime}$ are manifolds $f, g, F, G$ differentiable maps, $f$ and $g$ are transversal and $Y^{\prime}=Y \times_{X} X^{\prime}, f$ and $F$ are equipped with compatible $\operatorname{spin}^{c}$ structures. If further we have supports $Z \subset X, Z^{\prime} \subset X^{\prime}, T \subset Y, T^{\prime} \subset Y^{\prime}$ with $Z^{\prime} \supset G^{-1}(Z), T^{\prime} \supset g^{-1}(T), T \supset f(Z), T^{\prime} \supset F\left(Z^{\prime}\right)$ then $\mathrm{G}^{-1} \mathrm{f}_{*}=\mathrm{F}_{*} \mathrm{~g}^{-1}: \mathrm{K}_{\mathrm{Z}}(\mathrm{Y}) \rightarrow \mathrm{K}_{\mathrm{T}}\left(\mathrm{X}^{\prime}\right)$.

Thus $\mathrm{f}_{*}$ is defined if $\mathrm{f}$ is a $\operatorname{spin}^{\mathrm{c}}$ immersion (as the composition of the Bott isomorphism of a tubular neighborhood, and the excision map). It is also defined if $\mathrm{f}$ is the projection $\mathrm{X}=\mathrm{Y} \times \mathrm{C}^{\mathrm{n}} \rightarrow \mathrm{Y}$ (with the canonical $\operatorname{spin}^{\mathrm{c}}$ structure), as the composition of the restriction map: $K_{Z}(X) \rightarrow K_{B}(X)$ where $B \supset Z$ is a ball bundle with basis $f(Z)$ and of radius some suitably large continuous function, and of the inverse of the Bott isomorphism $\mathrm{K}_{\mathrm{B}}(\mathrm{X}) \rightarrow \mathrm{K}_{\mathrm{f}(\mathrm{Z})}(\mathrm{Y})$ (this is well defined because the pair $(\mathrm{X}, \mathrm{f}(\mathrm{Z}))$ can be deformed in the pair $(\mathrm{X}, \mathrm{B}))$. In the general case $\mathrm{f}$ is the composition of two such maps.

In particular if $X$ has a $\operatorname{spin}^{\mathrm{c}}$ structure (eg. a complex or a symplectic structure), and $\mathbf{Z} \subset \mathbf{X}$ is compact, the character $\chi^{\mathrm{top}:} \mathrm{K}_{\mathrm{Z}}(\mathrm{X}) \rightarrow \mathbf{Z}=\mathrm{K}$ (point) is the push-forward map by the $\operatorname{spin}^{\mathrm{c}}$ map $\mathrm{X} \rightarrow$ point.

2 This follows from the fact that if $\mathrm{N}$ and $\mathrm{N}^{\prime} \subset \mathrm{N}$ are equipped with $\operatorname{spin}^{\mathrm{c}}$ strutures, and we set $\mathrm{N}^{\prime \prime}=\mathrm{N}^{\prime} \perp$, then

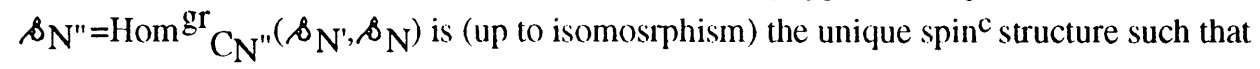
$\left(\mathrm{N}, \S_{\mathrm{N}}\right)=\left(\mathrm{N}^{\prime}, \aleph_{\mathrm{N}^{\prime}}\right) \oplus\left(\mathrm{N}^{\prime \prime}, \aleph_{\mathrm{N}^{\prime \prime}}\right)$. This complementation property does not hold for complex structures. 


\section{Appendix 2. D-Modules}

In the introduction we mentionned that the natural framework to describe linear differential systems is the theory of $\mathscr{D}$-modules. This section gives a résumé of the basic definitions and constructions concerning $\mathscr{D}$-modules. For further details see the book of Sato-Kawai-Kashiwa, or Borel et al seminar (in the algebro-geometric setting), and the books of Kashiwara 5, Schapira, and Björk, or the Grenoble seminar (Boutet de MonvelLejeune-Malgrange, 1965). Many operations on $\mathscr{D}$-modules are a superposition of several manipulations and are suitably described in terms of derived categories; for this we refer to the same, and Verdier, Borel et al 2, Kashiwara-Schapira 2.

a. Introduction Let $\mathrm{X}$ be an analytic manifold. We denote by $\mathcal{O}$, the sheaf of complex valued analytic functions, $\Omega$ the sheaf of differential forms of maximum degree (densities) and $\mathscr{D}$ the sheaf of analytic differential operators: $\mathcal{O}$ is a left $\mathscr{D}$-module and $\Omega$ a right $\mathscr{D}$-module. $\mathscr{D}$ has a canonical filtration $\left(\mathscr{D}_{\mathrm{m}}\right.$ is the sub-sheaf of operators of degree $\left.\leq \mathrm{m}\right)$ and $\operatorname{gr} \mathscr{D}$ identifies with the sheaf of sections of the symmetric algebra STX (equivalently the graded sheaf of polynomial functions on $T^{*} \mathrm{X}$, with coefficients in $\mathscr{O}_{\mathrm{X}}$ ). $\mathscr{D}$ is coherent (because $\mathrm{gr} \mathscr{D}$ is). A system of differential equations on $\mathrm{X}$ is often described as a complex of differential operators

$$
\mathrm{p}: \ldots \mathrm{E}^{\mathrm{k}} \stackrel{\mathrm{P}_{\mathrm{k}}}{\rightarrow} \mathrm{E}^{\mathrm{k}+1} \rightarrow \ldots
$$

where the $E^{k}$ are analytic vector bundles on $X$ (here we denote by the same letter the sheaf of analytic sections of $E$ ), and where we are interested in the solution sheaves, ie. the cohomology sheaves of $\mathrm{p} .{ }^{3}$ One is also interested by the solutions with $\mathrm{C}^{\infty}$ or distribution coefficients, or more generally with coefficients in a sheaf $\mathrm{N}$ of left $\mathscr{D}$-modules.

A more systematic description consists in defining a differential system $\mathrm{P}$ as a complex of right $\mathscr{D}$-modules (or rather an object of the derived category of these) ${ }^{4}$; the sheaf of solutions with coefficients in $\mathrm{N}$ is the complete tensor product

$$
\operatorname{Sol}(\mathrm{P}, \mathrm{N})=\mathrm{P} \otimes_{\mathscr{D}}^{\mathrm{L}} \mathrm{N}
$$

\footnotetext{
${ }^{3}$ For example if $\mathrm{p}: \mathrm{E}^{0} \rightarrow \mathrm{E}^{1}$ is of length 2, the homology sheaf $\mathrm{H}^{0}$ is the sheaf of solutions of the equation $\mathrm{pf}=0$, and $\mathrm{H}^{1}$ is the sheaf of obstructions to solving the equation $\mathrm{pf}=\mathrm{g}$.

${ }^{4}$ the sheaf of right $\mathscr{D}$-modules associated to $\mathrm{p}(\mathrm{A} 3)$ is $\mathrm{P}^{\mathrm{d}}: \cdots \rightarrow \mathscr{E}^{\mathrm{k}} \rightarrow \mathscr{E}^{\mathrm{k}+1} \rightarrow \cdots$ with $\mathscr{E}^{\mathrm{k}}=\operatorname{Diff}\left(\mathcal{O}, \mathrm{E}^{\mathrm{k}}\right)$ the sheaf of differential operators of type $\mathcal{O} \rightarrow E^{k}$, and differential $Q \rightarrow p_{0} Q$, so that $P^{d} \otimes \mathcal{O}$ is the initial complex $\mathrm{p}$. Similarily one associates to $\mathrm{p}$ the complex $\mathrm{P} g$ of left $\mathscr{D}$-modules $\operatorname{Diff}(\mathrm{E}, \mathcal{O})$, for which $\operatorname{Sol}(\mathrm{P})=\operatorname{Hom}_{\mathscr{D}}(\mathrm{Pg}, \mathcal{O})$.
} 
which is an object of the derived category of sheaves. Familiar systems correspond to coherent $\mathscr{D}$-modules (or complexes with coherent cohomology). In fact we will be mostly dealing with $\mathscr{D}$-modules which possess good filtrations. ${ }^{5}$

\section{b. Characteristic set}

In general, if $\mathrm{M}$ is a left (or right) $\mathscr{D}_{\mathrm{X}}$-module, the characteristic set, or analytic microsupport SS $M$ is the conic set $\operatorname{supp}\left(\mathscr{E} \otimes_{\mathrm{p}^{-1}} \mathrm{p}^{-1} \mathrm{M}\right) \subset \mathrm{T}^{*} \mathrm{X}$, where $\mathrm{p}$ is the projection $\mathrm{T}^{*} \mathrm{X} \rightarrow \mathrm{X}$ and $\mathscr{E}$ is the sheaf on $\mathrm{T}^{*} \mathrm{X}$ of analytic pseudo-differential operators. This definition extends to complexes or objects of the derived category. If $\mathrm{M}$ is coherent (or has coherent cohomology), SS M is also the support of gr M for any good filtration, and it is an analytic involutive subset of $T^{*} X^{6}$ (cf the book of Sato, Kawai, Kashiwara).

\section{c. Direct and inverse image}

Let $X, Y$ be analytic manifolds and $f: X \rightarrow Y$ an analytic map. We define the transfer module $\mathscr{D}_{\mathrm{X} \rightarrow \mathrm{Y}}$ as the sheaf on $\mathrm{X}$ of $\mathrm{f}$-differential operators of type $\mathcal{O}_{\mathrm{Y}} \rightarrow \mathcal{O}_{\mathrm{X}}$ (local sections are operators of the form $u \in \mathscr{O}_{\mathrm{Y}} \rightarrow \mathrm{PX}_{\mathrm{X}}\left(\mathrm{Q}_{\mathrm{Y}} \mathrm{u}_{\mathrm{O}} \mathrm{f}\right)$ with $\left.\mathrm{PX}_{\mathrm{X}} \in \mathscr{D}_{\mathrm{X}}, \mathrm{Q}_{\mathrm{Y}} \in \mathscr{D}_{\mathrm{Y}}\right) . \mathscr{D}_{\mathrm{X} \rightarrow \mathrm{Y}}$ is a left $\mathscr{D}_{\mathrm{X}}$-module and a right $\mathrm{f}^{-1} \mathscr{D}_{\mathrm{Y}}$-module. Similarily one defines the transfer module $\mathscr{D}_{\mathrm{Y} \leftarrow \mathrm{X}}$ as the sheaf on $\mathrm{X}$ : it is the sheaf of $\mathrm{f}$-differential operators of type $\Omega_{\mathrm{Y}} \rightarrow \Omega_{\mathrm{X}}$, a $\left(\mathrm{f}^{-1} \mathscr{D}_{\mathrm{Y}}, \mathscr{D}_{\mathrm{X}}\right)$ bimodule.

Example1 - If $\mathrm{f}$ is submersive we have the relative De Rham complex $\mathrm{d}_{\mathrm{X} / \mathrm{Y}}$ :

$$
\mathrm{d}_{\mathrm{X} / \mathrm{Y}}: 0 \rightarrow \mathcal{O}_{\mathrm{Y}} \rightarrow \mathrm{T} * \mathrm{X} / \mathrm{Y} \rightarrow \cdots \Lambda^{\mathrm{k}} \mathrm{T} * \mathrm{X} / \mathrm{Y} \rightarrow \Lambda^{\mathrm{k}+1} \mathrm{~T} * \mathrm{X} / \mathrm{Y} \rightarrow \cdots
$$

As a left $\mathscr{D}_{\mathrm{X}}$-module, $\mathscr{D}_{\mathrm{X} \rightarrow \mathrm{Y}}$ is generated by the pullback operator $\varepsilon\left(\mathrm{u} \rightarrow \mathrm{u}_{\mathrm{o}} \mathrm{f}\right)$; it is a flat $\mathrm{f}^{-1} \mathscr{D}_{\mathrm{Y}}$-module (as $\mathcal{O}_{\mathrm{X}}$ is flat on $\mathrm{f}^{-1} \mathcal{O}_{\mathrm{Y}}$ ); and the complex $\mathrm{DR}_{\mathrm{X} / \mathrm{Y}}^{\mathrm{g}}$ of left $\mathscr{D}$-modules associated to $d_{X / Y}$ is a locally free resolution of $\mathscr{D}_{X \rightarrow Y}$ (the augmentation is $P \in \mathscr{D}_{X} \rightarrow P_{0} \varepsilon$ ).

If $\mathrm{f}: \mathrm{X} \rightarrow \mathrm{Y}$ is an immersion we have $\mathscr{D}_{\mathrm{X} \rightarrow \mathrm{Y}} \simeq \mathcal{O}_{\mathrm{X}} \otimes_{\mathrm{f}-1 \mathscr{O}_{\mathrm{Y}}} \mathrm{f}^{-1} \mathscr{D}_{\mathrm{Y}}$. It is a locally free $\mathscr{D}_{\mathrm{X}^{-}}$ module; locally $\mathrm{f}$ is isomorphic with the zero section of a vector bundle, whose Koszul complex defines (extending coefficients to $\mathscr{D}_{\mathrm{Y}}$ ) a locally free resolution of $\mathscr{D}_{\mathrm{X} \rightarrow \mathrm{Y}}$ on $\mathscr{D}_{\mathrm{Y}}$.

\footnotetext{
5 A good filtration on a $\mathscr{D}$-module $M$ is a filtration $M=U M_{k}$, with $M_{k} \mathscr{O}_{X}$ cohérent, $M_{k}=0$ if $k \ll 0, M_{k} \mathscr{D}_{p} \subset$ $\mathbf{M}_{\mathbf{k}+\mathrm{p}}$, with equality if $\mathrm{k} \gg 0$. A $\mathscr{D}$-module is coherent iff it possesses locally good filtrations. Algebraic coherent $\mathscr{D}$-modules and holonomic $\mathscr{D}$-modules possess global good filtrations but in general existence of a globally defined good filtration on a coferent $\mathscr{D}$-module is not known. The canonical flitration of $\mathscr{D}$ is a good filtration.

${ }^{6}$ ie. if $\mathrm{f}$ and $\mathrm{g}$ vanish on char $\mathcal{M}$ then so does their Poisson bracket $\{\mathrm{f}, \mathrm{g}\}=\Sigma \partial \mathrm{f} / \partial \xi_{\mathrm{j}} \partial \mathrm{g} / \partial \mathrm{x}_{\mathrm{j}}-\partial \mathrm{f} / \partial \mathrm{x}_{\mathrm{j}} \partial \mathrm{g} / \partial \xi_{\mathrm{j}}$ (in any system of local coordinates)
} 
If $\mathscr{M}$ is a right $\mathscr{D}_{\mathrm{X}}$-module (resp. a left $\mathscr{D}_{\mathrm{Y}}$-module) the direct image $\mathrm{f}_{+} \mathrm{M}$ is the object of the derived category of right $\mathscr{D}_{\mathrm{Y}^{-}}$-modules defined by

$$
\mathrm{f}_{+} \mathrm{M}=\mathrm{Rf}_{*}\left(\mathrm{M}^{\mathrm{L}} \mathscr{D}_{\mathrm{X}} \mathscr{D}_{\mathrm{X} \rightarrow \mathrm{Y}}\right) \in \mathrm{obD}^{\mathrm{b}}\left(\mathscr{D}_{\mathrm{Y}}\right)
$$

(resp. the inverse image $f^{+} M$ is

$$
\left.\mathrm{f}^{+} \mathrm{M}=\mathrm{f}^{-1}(\mathrm{M}) \stackrel{L}{\otimes} \mathscr{D}_{\mathrm{Y}}\right) \mathscr{D}_{\mathrm{Y} \leftarrow \mathrm{X}}[-\mathrm{d}] \in \mathrm{obD}^{\mathrm{b}}\left(\mathscr{D}_{\mathrm{Y}}\right) \quad(\mathrm{d}=\operatorname{dim} \mathrm{X}-\operatorname{dim} \mathrm{Y})
$$

one defines similarily the direct image of a left $\mathscr{D}_{\mathrm{X}}$-module or a right $\mathscr{D}_{\mathrm{Y}}$-module using the transfer module $\mathscr{D}_{\mathrm{Y} \leftarrow \mathrm{X}}$ ).

Example 2 - If $\mathrm{f}: \mathrm{X} \rightarrow \mathrm{Y}$ is a closed immersion, the direct image $\mathrm{f}_{+}$is defined for $\mathscr{D}$-modules: the functor $\mathrm{M} \rightarrow \mathrm{f}_{*} \mathrm{M} \otimes_{\mathscr{D}_{\mathrm{X}}} \mathscr{D}_{\mathrm{X} \rightarrow \mathrm{Y}}$ is exact; it is a category equivalence (Kashiwara equivalence) between $\mathscr{D}_{\mathrm{X}}$-modules and $\mathscr{D}_{\mathrm{Y}}$-modules "algebraically" supported by $\mathrm{X}$ (ie. any of whose sections is killed by some power of the ideal of $\mathrm{X}$ ).

Dually if $\mathrm{f}: \mathrm{X} \rightarrow \mathrm{Y}$ is a submersion with cohomologically trivial fiber - eg. $\mathrm{X}$ is the germ of some manifold along a continuous section of $\mathrm{f}$ - it is shown in Boutet de MonvelMalgrange that the inverse image $\mathrm{f}^{+}$realizes a category equivalence between coherent $\mathscr{D}_{\mathrm{Y}^{-}}$ modules and the category of coherent $D_{\mathrm{X}}$-modules which are regularily characteristic along horizontal covectors of the submersion (the characteristic set of $d_{X / Y}$ ). This is particularily useful in the following case: $Y$ is a complex manifold, $X=Y_{R}$ the real sublying manifold germ of $\mathrm{Y} \times \overline{\mathrm{Y}}$ along the diagonal, and $\mathrm{f}: \mathrm{X} \rightarrow \mathrm{Y}$ is the canonical projection.

\section{d. Symbol, K-theoretical element associated to a $\mathscr{D}$-module}

Let $\mathrm{X}$ be a complex manifold, and $\mathrm{M}$ a $\mathscr{D}$-module possessing a good filtration. If $\mathrm{Z} \subset \mathrm{T}^{*} \mathrm{X}$ is a closed conic set containing SSM we define:

$$
[\mathrm{M}]_{Z}^{\mathrm{an}} \in \mathrm{K}_{\mathrm{Z}}^{\mathrm{an}}(\mathrm{T} * \mathrm{X})
$$

the element of the Grothendieck group of homogeneous sheaves on $T^{*} X$. This element (or rather its restriction to any subset with compact basis in $\mathrm{T}^{*} \mathrm{X}$ ) does not depend on the choice of a good filtration on $\mathrm{M}$.

We also define the topological symbol

$$
[\mathrm{M}]_{\mathrm{Z}}^{\mathrm{top}} \in \mathrm{K}_{\mathrm{Z}}(\mathrm{T} * \mathrm{X})
$$

of right $\mathscr{D}$-modules possessing good filtrations (or similarily for a left $\mathscr{D}$-module) by the following axioms: 
(i) it is additive for exact sequences ie. $[M]_{Z}^{\text {top }}=\left[M^{\prime}\right]_{Z}^{\text {top }}+\left[M^{\prime \prime}\right]_{Z}^{\text {top }}$ if there exists an exact sequence $0 \rightarrow \mathrm{M}^{\prime} \rightarrow \mathrm{M} \rightarrow \mathrm{M}^{\prime \prime} \rightarrow 0$

(ii) if $\mathrm{M}$ corresponds (as above) to a complex $\mathrm{P}$ of differential operators whose symbol $\sigma(\mathrm{P})$ is exact outside of $\mathrm{Z}$, then $[\mathrm{M}]_{\mathrm{Z}}^{\mathrm{top}}=[\sigma(\mathrm{P})]_{\mathrm{Z}}$ (the element of $\mathrm{K}_{\mathrm{Z}}\left(\mathrm{T}^{*} \mathrm{X}\right)$ defined by $\sigma(\mathrm{P})$ ).

(iii) it is compatible with submersive inverse images. More precisely let $\mathrm{f}: \mathrm{X} \rightarrow \mathrm{Y}$ be a submersion. If $\mathrm{M}$ is a coherent $\mathscr{D}$-module (possessing a good filtration) we have $\mathrm{f}^{+} \mathrm{M}=$ $\mathrm{f}^{-1} \mathrm{M} \otimes_{\mathrm{f}^{-1} \mathscr{D}} \mathscr{D}_{\mathrm{Y} \leftarrow \mathrm{X}}[\mathrm{d}](\mathrm{d}=\operatorname{dim} \mathrm{X} / \mathrm{Y})$. Denote $\mathrm{F}: \mathrm{f}^{-1} \mathrm{~T}^{*} \mathrm{Y}=\mathrm{X} \times_{\mathrm{Y}} \mathrm{T}^{*} \mathrm{Y} \hookrightarrow \mathrm{T}^{*} \mathrm{X}$ the cotangent map: its image is $\mathrm{H}=\mathrm{car} \mathscr{D}_{\mathrm{Y} \leftarrow \mathrm{X}} \subset \mathrm{T}^{*} \mathrm{Y}$, the set of horizontal covectors; denote $\overline{\mathrm{f}}$ : $\mathrm{f}^{-1} \mathrm{~T}^{*} \mathrm{Y} \rightarrow \mathrm{T}^{*} \mathrm{Y}$ the projection. Then we have car $\left(\mathrm{f}^{+}(\mathrm{M})\right)=\mathrm{F}^{-1}(\mathrm{car} \mathrm{M}) \subset \mathrm{H}$, and

$$
\left[\mathrm{f}^{+} \mathrm{M}\right]_{\mathrm{f}^{+} \mathrm{Z}}^{\mathrm{top}}=\overline{\mathrm{f}}^{-1}[\mathrm{M}]_{\mathrm{Z}}^{\mathrm{top}} \cdot\left[\mathscr{D}_{\mathrm{X} \leftarrow \mathrm{Y}}[\mathrm{d}]\right]_{\mathrm{Z}^{\prime}}^{\text {top }}=\mathrm{F}_{*} \overline{\mathrm{f}}^{-1}[\mathrm{M}]_{\mathrm{Z}}^{\mathrm{top}}(\mathrm{d}=\operatorname{dim} \mathrm{X} / \mathrm{Y})
$$

the $\mathrm{K}$-theoretical image. These axioms define $[\mathrm{M}]_{Z}^{\text {top }}$ if $\mathrm{X}$ is the germ of a complex manifold along a compact set, or if $X$ is a projective manifold, since $M$ then possesses a "good" locally free resolution (corresponding to a locally free resolution or grM), whose symbol defines $[M]_{Z}^{\text {top }}$. In the general case the real sublying manifold $X_{R}$ is Stein, so $\left[M_{R}\right]_{Z}^{\text {top }}$ is well defined, so as $[\mathrm{M}]_{\mathrm{Z}}^{\mathrm{top}}$ since in this case the $\mathrm{K}$-theoretical map $\mathrm{F}_{*} \mathrm{f}^{-1}: \mathrm{K}_{\mathrm{Z}}\left(\mathrm{T}^{*} \mathrm{X}\right) \rightarrow \mathrm{K}_{\mathrm{fZ}}\left(\mathrm{T}^{*} \mathrm{X}_{\mathrm{R}}\right)$ is one to one (it is the Bott isomorphism). Reducing similarily to the case where $\mathrm{X}$ is real one shows that the symbol $[\mathrm{M}]_{\mathrm{Z}}^{\mathrm{top}}$ is well behaved under closed immersions:

(iv) If $\mathrm{f}: \mathrm{X} \rightarrow \mathrm{Y}$ is a closed immersion, $\mathrm{M}$ a $\mathscr{D}_{\mathrm{X}}$-module with a good filtration and car $\mathrm{M}=$ Z. Then $f_{+} M$ possesses a good filtration, we have car $f_{+} M=\bar{f} F^{-1}(Z)$, and

$$
\begin{gathered}
{\left[\mathrm{f}_{+} \mathrm{M}\right]_{\mathrm{Z}^{\prime}}^{\mathrm{top}}=\overline{\mathrm{f}}_{*} \mathrm{~F}^{-1}[\mathrm{M}]_{\mathrm{Z}}^{\mathrm{top}} \quad\left(\mathrm{K} \text {-theoretical image (with } \overline{\mathrm{f}}: \mathrm{f}^{-1}\left(\mathrm{~T}^{*} \mathrm{Y}\right) \rightarrow \mathrm{T}^{*} \mathrm{Y}\right. \text { the }} \\
\text { projection, and } \mathrm{F}: \mathrm{f}^{-1}\left(\mathrm{~T}^{*} \mathrm{Y}\right) \rightarrow \mathrm{T}^{*} \mathrm{X} \text { the cotangent map) }
\end{gathered}
$$

Remark - if $m$ is a coherent $0_{\mathrm{X}}$-module with support $\subset \mathrm{Z}$, we set note $[m]_{\mathrm{Z}}^{\text {top }} \in \mathrm{K}_{\mathrm{Z}}(\mathrm{X})$ whose inverse image on $T^{*} X$ is $\left[m \otimes_{\mathcal{O}_{X}} D_{X}\right]_{p^{-1}}^{t o p} \in K_{p^{-1}}\left(T^{*} X\right)$. This definition commutes with submersions and closed immersions. ${ }^{7}$ This definition of course coincides with that of Baum, Fulton, Mac-Pherson when it is defined (eg. if $\mathrm{X}$ is a Stein or projective manifold so $m$ has finite locally free resolutions). In their work Baum, Fulton, Mac-Pherson prove the result for immersions by "deformation to the normal cone".

7 The canonical map $K_{Z}^{\text {an }}(X) \rightarrow K_{Z}^{\text {top }}(X)$ was defined by Baum-Fulton-Mac Pherson when $X$ is a projective space, using a deformation to the normal cone. Our definition uses the real sublying manifold $X_{R}$ so $X$ does not needs to be projective. Note that going from $X$ to $X_{R}$, one looses nothing at the level of $\mathscr{D}$-modules, but one looses all modular or continuous information contained in $\mathrm{K}_{\mathrm{Z}}^{\mathrm{an}}(\mathrm{X})$. 


\section{Appendix 3. Sketch of the proof of the index formula}

The data for the index theorem consists in a complex manifold $X$, a system of differential equations on $\mathrm{X}$ described by a coherent right $\mathscr{D}$-module $\mathrm{M}$ (possessing a good filtration), and a sheaf of coefficients $\mathrm{N}$ (a left $\mathscr{D}$-module).

In what follows $\mathrm{N}$ will always be of the form $\mathrm{N}=\mathcal{O}_{\mathrm{U}}$ where $\mathrm{U}$ is a real analytic submanifold with boundary (or corners). ${ }^{8}$ The microsupport SS N=SS U is then the set of all "outgoing" normal covectors, ie complex covector $\zeta$ such that $\operatorname{Re} \zeta$ is negative on the 1 st order jet of $U$ (this makes sense unambiguously if $M$ is a real analytic submanifold with corners).

example - if $\mathrm{U}$ is a totally real submanifold in $\mathrm{X}$ (real case), SSU is the set of pure imaginary covectors at points of $U$.

If $U$ is a submanifold of real dimension $2 \operatorname{dim} X$, with boundary $\partial U$ a real-analytic

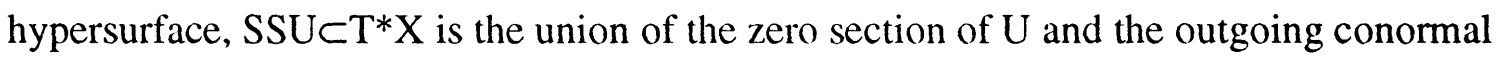
bundle of $\partial \mathrm{U}$ (set of all $\zeta$ at points.

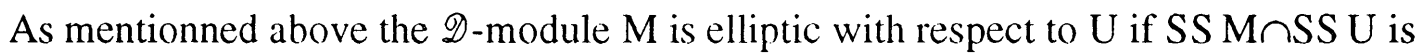

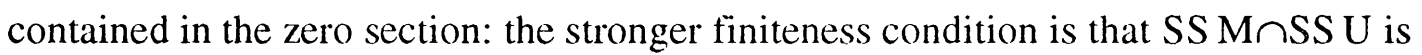
compact. We then dispose of the following objects:

- the characteristic set $\mathrm{Z}=\mathrm{SS} \mathrm{M}$, and the symbol $[\mathrm{M}]_{\mathrm{Z}}^{\mathrm{top}} \in \mathrm{K}_{\mathrm{Z}}\left(\mathrm{T}^{*} \mathrm{X}\right)^{9}$

- the microsupport SSN: in the case considered here $\left(\mathrm{N}=\mathcal{O}_{\mathrm{U}}\right)$ this always has a tubular neighborhood with a canonical $\operatorname{spin}^{c}$ structure, and by definition $[\mathrm{N}]$ is the Bott element corresponding to this. ${ }^{10}$

Thus the terms in the index formula (5) are well defined. The main idea of the proof is to embed everything in a numeric space where the formula is known. However ellipticity is not preserved by closed embeddings (a system whose solutions are carried by a proper submanifold cannot be elliptic), so it is useful to slightly enlarge the definition. In the cases we are dealing with ( $\mathrm{U}$ a real analytic manifold with corners) it is easy to see that $U$ can be

\footnotetext{
${ }^{8}$ the case where $\mathrm{N}$ is a coherent left $\mathscr{D}$-module can formally be reduced to the case $\mathrm{M}=\mathcal{O}$. There clearly should be a more general case, making the symmetry between $\mathrm{M}$ and $\mathrm{N}$ more apparent, but the $\mathrm{K}$-theoretical aspect in more general cases remains to be developped. As mentionned above the case where $\mathrm{N}$ is associated to a constructible sheaf on $\mathrm{X}$ has been examined by Schapira and Schneiders.

${ }^{9}$ this is really only defined above compact subsets of X, but this is enough for the index formula where it only needs to be defined dear the compact set SS M $\cap S S$ U.

10 for example if $U$ is an open subset with analytic boundary, SS U can be deformed into the zero section; for the zero section the tubular neighborhood is $\mathrm{T}^{*} \mathrm{X}$, which is a complex vector bundle. The $\mathrm{K}$-theoretical element [N] for more generent coefficient sheaves remains to be constructed.
} 
deformed and thickened in the following sense: there existes a continuous one parameter family $U_{\varepsilon}$ of neighborhoods of $U$, such that each $U_{\varepsilon}$ is a manifold with boundary, $U_{\varepsilon} \subset U_{\varepsilon^{\prime}}$ if $\varepsilon<\varepsilon^{\prime}$, and $S S U_{\varepsilon} \rightarrow S S U$ if $\varepsilon \rightarrow 0$. We will say that such a family of neighborhoods is adapted to $\mathrm{U}$, a use the following generalization of ellipticity:

Définition A3 - $\mathrm{M}$ is almost elliptic with respect to $U$ if there exists an adapted family $U_{\varepsilon}$ of neighborhoods such that $M$ is adapted with respect to $U_{\varepsilon}$ for small $\varepsilon$.

examples : an elliptic module is almost elliptic. Products of almost elliptic modules are almost elliptic. If $M$ is almost elliptic with respect to $U$, and $f$ is an analytic embedding, $f_{+} M$ is almost elliptic with respect to $\mathrm{f}(\mathrm{U})$. Finally a holonomic module is always almost elliptic, with respect to any $U$.

The index formula extends naturally to almost elliptic systems (replacing the product $[\mathrm{M}]_{\mathrm{CarM}}^{\mathrm{top}}\left[\mathcal{O}_{\mathrm{U}}\right]_{\mathrm{SSU}}$ (or the restriction $[\mathrm{M}]_{\mathrm{carM}}^{\mathrm{top}} \mid \mathrm{SSU}$ ) by the limit of the deformations $\left.[\mathrm{M}]_{\mathrm{carM}}^{\text {top }}\left[{ }^{\circ}{ }_{\mathrm{U}_{\varepsilon}}\right]_{S S U_{\varepsilon}}\right)$, and we prove it in this framework, which allows embeddings.

The index theorem may then be proved as follows:

1. We first replace the manifold $X$ by the sublying real manifold $X_{R}$, and $M$ by $M_{R}$ 2. The choose a closed immersion $f$ to embed everything in a numeric space $\mathbf{R}^{\mathbf{n}}$, and possibly thicken in $\mathbf{C}^{\mathrm{n}}$ to reduce to the case where $U$ is a small ellipsoïd neighborhood of the real unit ball; in this cas the formula is already established: it reduces to the index formula for Toeplitz operators on a ball and is a particular case of the Bott periodicity theorem.

The topological character $\chi^{\text {top }}$ or the K-theoretical push-forward were precisely constructed to follow in these operations.

As was shown by Atiyah-Segal, the absolute index formula has a natural generalization to systems to systems depending on parameters. ${ }^{11}$ In our analytic framework these are described as follows: we first have an analytic map $f: X \rightarrow Y$ (of real or complex manifolds), a complex $\mathrm{M}$ of right $\mathscr{D}_{\mathrm{X} / \mathrm{Y}^{-}}$-modules (with good filtrations), representing an analytic family of differential systems on the fibers, and a sheaf of coefficients $\mathrm{N}=\mathrm{O}_{\mathrm{U}}$, associated as above to a real analytic submanifold with corners $U \subset X$. The relative characteristic variety $\mathrm{Z}=\mathrm{car} \mathrm{M} / \mathrm{Y} \subset \mathrm{T}^{*} \mathrm{X} / \mathrm{Y}$ is the support of $\mathrm{grM}$ (for any good filtration). To $M$ we associate the symbol

$$
[\mathrm{M}]_{\mathrm{Z}}^{\mathrm{top}} \in \mathrm{K}_{\mathrm{Z}}\left(\mathrm{T}^{*} \mathrm{X} / \mathrm{Y}\right)
$$

\footnotetext{
11 also to G-equivariant systems, $\mathrm{G}$ a compact group (the index is then a virtual representation of $\mathrm{G}$ ), to which case our proof adapts easily - using equivariant embeddings.
} 
defined as above (it is only defined above compact subsets of $\mathrm{X}$ ). The direct image of MIU (describing solutions along fibers of $U$ ) is $f_{+} M=R f_{*}\left(M \stackrel{L}{\otimes}_{\mathscr{D}} N\right)$. To $N$ we associate its relative microsupport $S S N \subset T^{*} X / Y$, whose fiber above $y \in Y$ is $S_{S U}$. The finiteness (ellipticity) condition is that the projection $S S M \cap S S N \rightarrow Y$ be proper, ie. that $M_{y}$ be elliptic along $U_{y}$ for all $y \in Y$ and this ensures that $f_{+} M$ has coherent cohomology. In that case the support of $\mathrm{f}_{+} \mathrm{M}$ is contained in the projection $\mathrm{Z}^{\prime}$ of $\mathrm{Z}=\mathrm{car} \mathrm{M} / \mathrm{Y} \cap \mathrm{SS} \mathrm{U} / \mathrm{Y}$, and the following formula is the natural generalization of the index formula:

$$
\left[\mathrm{f}_{+} \mathrm{M}\right]_{\mathrm{Z}^{\mathrm{p}}}^{\mathrm{top}}=\overline{\mathrm{f}}_{*}\left([\mathrm{M}]_{\mathrm{Z}}^{\mathrm{top}}\left[\mathrm{N}_{\mathrm{U}}\right]\right) \in \mathrm{K}_{\mathrm{Z}}(\mathrm{Y}) \quad \text { (K-theoretical image) }
$$

where $\bar{f}$ is the projection $T^{*} X / Y \rightarrow Y$. The proof is an immediate adaptation of the proof sketched above.

\section{Relative index theorem}

We end these notes by a brief description of the relative case. In the relative index formula we are given an analytic map $\mathrm{f}: \mathrm{X} \rightarrow \mathrm{Y}$ between analytic manifolds, a $\mathscr{D}$-module $\mathrm{M}$ on $X$, and a subset $U$ defining the sheaf of coefficients. We are interested in the direct image $\mathrm{f}_{+}(\mathrm{M} \mid \mathrm{U})$ : a relative ellipticity condition will ensure that this is coherent, and the relative index formula will then describe its symbol bundle $\left[\mathrm{f}_{+}(\mathrm{M} \mid \mathrm{U})\right]$ in terms of $[\mathrm{M}]$.

Let us describe this more precisely. First the relative index formula below, as all formulas above, is compatible with closed immersions: replacing $\mathrm{X}$ by $\mathrm{X} \times \mathrm{Y}, \mathrm{M}$ and $\mathrm{U}$ by their direct image by the graph map Id $\times f$, and $f$ by the projection $X \times Y \rightarrow Y$, we are reduced to the case where $\mathrm{f}$ is submersive (a projection), which we will always suppose from now on. As above we denote

$$
\begin{array}{ll}
\mathrm{H}=\mathrm{X} \times \mathrm{Y}^{*} \mathrm{Y} & \\
\overline{\mathrm{f}}: \mathrm{H} \rightarrow \mathrm{T}^{*} \mathrm{Y} & \text { the second projection } \\
\mathrm{F}: \mathrm{H} \rightarrow \mathrm{T}^{*} \mathrm{X} & \text { the cotangent map }
\end{array}
$$

to our set $\mathrm{U}$ defining the coefficient sheaf we associate the transfer module $\mathscr{D}_{\mathrm{U} \rightarrow \mathrm{Y}}=$ $\mathbf{C}_{\mathrm{U}} \otimes \mathscr{D}_{\mathrm{X} \rightarrow \mathrm{Y}}$. We define the microsupport SS $\mathscr{D}_{\mathrm{U} \rightarrow \mathrm{Y}}=\mathrm{SSC}_{\mathrm{U}}+\mathrm{SS} \mathscr{D}_{\mathrm{X} \rightarrow \mathrm{Y}}$ (the set of all covector of the form $\xi+\eta$ with $\xi \in S S U$ and $\eta \in H=S S D_{X \rightarrow Y}$ at points of $U$ ). We denote further

$$
\begin{aligned}
& \mathrm{U}_{\mathrm{e}}=\mathrm{SS} \mathscr{D}_{\mathrm{U} \rightarrow \mathrm{Y}}=\mathrm{SSU} \times_{\mathrm{X}} \mathrm{H} \\
& \mathrm{F}_{\mathrm{e}}: \mathrm{U}_{\mathrm{e}} \rightarrow \mathrm{T}^{*} \mathrm{X} \text { the map which extends } \mathrm{F} \text { by } \mathrm{F}_{\mathrm{e}}(\eta, \mathrm{h})=\eta+\mathrm{h} \\
& \overline{\mathrm{f}}_{\mathrm{e}}: \mathrm{U}_{\mathrm{e}} \rightarrow \mathrm{T}^{*} \mathrm{Y} \text { the projection }
\end{aligned}
$$


The relative ellipticity ellipticity condition is the following: we have seen above the definition of ellipticity with respect to $U$ for a vertical $D_{X / Y}$-module, which is the straightforward generalization of the ellipticity condition in the absolute case. The $\mathrm{M}$ is relatively elliptic if locally (near any point of $\partial U$ ) it is a quotient of a $\mathscr{D}$-module of the form $\mathrm{N} \otimes_{\mathscr{D}_{\mathrm{X} / \mathrm{Y}}} \mathscr{D}_{\mathrm{X}}$ for some coherent $\mathscr{D}_{\mathrm{X} / \mathrm{Y}}$-module $\mathrm{N}$, elliptic with respect to $\mathrm{U} .{ }^{12}$ Relative ellipticity implies the following geometric condition:

$$
\text { SS M and SS } \left.\mathscr{D}_{\mathrm{U} \rightarrow \mathrm{Y}} \text { meet along } \mathrm{H} \text { ("the zero section of } \mathrm{T}^{*} \mathrm{H} / \mathrm{T}^{*} \mathrm{Y}\right) \text {. }
$$

The finiteness condition is this relative ellipticity condition, plus the condition that the projection: $S S \mathrm{M} \cap S S \mathscr{D}_{\mathrm{U} \rightarrow \mathrm{Y}} \rightarrow \mathrm{T}^{*} \mathrm{Y}$ is proper. There is also a notion of almost ellipticity. However the relative ellipticity condition is more complicated than in the absolute case; it cannot be read on the principal symbol of operators alone and is harder to manipulate (and less stable).

Under this relative ellipticity and finiteness condition, it was shown by HouzelSchapira that the direct image $\mathrm{f}_{+} \mathrm{M}_{U}=\mathrm{Rf}_{*}\left(\mathrm{M}^{\otimes} \otimes_{\mathscr{D}} \mathscr{D}_{\mathrm{U} \rightarrow \mathrm{Y}}\right)$ is coherent and its characteristic set is contained in the set $Z^{\prime}=\bar{f}\left(S S M \cap S S \mathscr{D}_{U \rightarrow Y}\right)$. The relative index formula of Boutet de Monvel-Malgrange is in this case the straightforward generalization of (5)bis:

$$
\left[\mathrm{f}_{+} \mathrm{M}_{U}\right]_{\mathrm{Z}}^{\mathrm{top}}=\overline{\mathrm{f}}_{\mathrm{e} *} \mathrm{~F}^{-1}[\mathrm{M}]_{\mathrm{Z}}^{\mathrm{top}}
$$

We refer to the paper of Boutet de Monvel-Malgrange for the proof of the relative index formula and give here only the briefest indication. As above one may simplify the situation replacing $M$ by $M_{R}$ and embedding in a numeric space; we may thus reduce to the case $U=Y \times Q_{\varepsilon}$ where $Q_{\varepsilon}$ is a fixed complex ellipsoïd, neighborhood of a real ball. In any case, it is practical to use a resolution of $M$ by "vertical" $D$-modules of the type $N \otimes_{\mathscr{D}} / \mathrm{D}_{\mathrm{X}}$, and make use of vertical filtrations, ie. double filtrations of the form $\mathrm{M}_{\mathrm{pq}}=\mathrm{M}_{\mathrm{p}} \mathscr{D}_{\mathrm{X} / \mathrm{Y}}^{\mathrm{q}}$. The graded object associated to this is a vertical $\mathscr{D}_{\mathrm{H} / \mathrm{T}^{*} \mathrm{Y}}$ - module to which we may apply the theory above (with parameters): its direct image is coherent, and it is the first term of a spectral sequence which converges to $\operatorname{grf}_{+} \mathrm{M}_{\mathrm{U}}$. There still remains some work to do to compare the K-theoretical element associated to the "vertical" graded object" gr ${ }^{\mathrm{v}} \mathrm{M}$, which lives on $\mathrm{T}^{*} \mathrm{H} / \mathrm{T}^{*} \mathrm{Y} \simeq \mathrm{H} \times_{\mathrm{Y}} \mathrm{T}^{*} \mathrm{Y}$ and $[\mathrm{M}]_{\mathrm{Z}}^{\mathrm{top}}$ which lives on $\mathrm{T}^{*} \mathrm{X}$ (in fact they are bot compared to their "cones" which live along the zero section of $\mathrm{H}$ in $\mathrm{H} \times \mathrm{T}^{*} \mathrm{Y}$ ) and check that they give the same element by the K-theoretical image

12 equivalently any sestion $\mathrm{s}$ of $\mathrm{M}$ at a point of $\partial \mathrm{U}$ is killed by a vertical operator $\mathrm{P} \in \mathscr{D}_{\mathrm{X} / \mathrm{Y}}$ non characteristic for $\partial \mathrm{U}$. 


\section{BIBLIOGRAPHY}

Atiyah M.F. - K-theory, Benjamin, Amsterdam.

Atiyah M.F., Bott R., - On the periodicity theorem for complex vector bundles. Acta Math. 112 (1964) 229-247.

Atiyah M.F., Bott R., Schapiro A. - Clifford modules. Topology 3, supplément (1964), 3-83.

Atiyah M.F., Hirzebruch F. - Vector bundles and homogeneous spaces, Diff. Geometry, Proc. Symp. Pure Math., Amer. Math. Soc., Providence (1961) 7-38.

Atiyah M.F., Segal G.B. - The index of elliptic operators II, Ann. Math. 87 (1968) 531-545.

Atiyah M.F., Singer I.M. 1- The index of elliptic operators on compact manifolds, Bull. Amer. Math. Soc. 69 (1963) 422-433.

2- The index of elliptic operators I, Ann. Math. 87 (1968) 484-530.

3- The index of elliptic operators III, Ann. Math. 91, 546-604.

4- The index of elliptic operators IV, Ann. Math. 92 (1970) 119-138.

Baum P., Fulton W., Mac Pherson R. - Riemann-Roch and topological K-theory for singular varieties. Acta Math. 143, n³-4, (1979) 155-192.

Bernstein I.M., Gelfand S.I. - Meromorphy of the function $\mathrm{P}^{\lambda}$, Funkc. Anal. i Prilozen 3 (1969) 84-85 \& Funct. Anal. appl. 3 (1969) 68-69.

Bernstein I.N. - Modules over rings of differential operators. An investigation of the fundamental solution of equations with constant coefficients. Funkc. Anal i Prilozen 5, 2,(1971) 1-16 \& Funct. Anal. appl. 5 (1971) 89-101.

Björk J.E. - Rings of Differential Operators. North Holland 1979.

Borel A. et al. 1.- Intersection cohomology. Progress in Math. $\mathrm{n}^{\circ}$ 50, Birkhäuser (1984). 2.- Algebraic D-modules, Perspect. in Math. ${ }^{\circ} 2$, Academic Press (1987).

Boutet de Monvel L. 1.- On the index of Toeplitz operators of several complex variables. Inventiones Math. 50 (1979) 249-272. cf. aussi Séminaire EDP 1979, Ecole Polytechnique.

2.- Systèmes presqu'elliptiques: une autre démonstration de la formule de l'indice. Astérisque 131 (1985) 201-216.

3.- The index of almost elliptic systems. E. de Giorgi Colloquium, Research notes in Math. 125, Pitman 1985, 17-29.

4.- Indice des systèmes différentiels. Cours C.I.M.E. Venise, Juin 1992. Lecture Notes in Math (à paraître)

Boutet de Monvel L., Lejeune M., Malgrange B. - Opérateurs différentiels et pseudodifférentiels. Séminaire, Grenoble 1975-76. 
Boutet de Monvel L., Malgrange B. - Le théorème de l'indice relatif, Ann. Scientifiques de l' E.N.S. 23 (1990), 151-192.

Boutet de Monvel L., Sjöstrand J. - Sur la singularité des noyaux de Bergman et de Szegö. Astérisque 34-35 (1976) 123-164.

Brylinski J.L., Dubson A., Kashiwara M. - Formule de l'indice pour les modules holonômes et obstruction d'Euler locale. C.R. Acad. Sci. 293 (1981) 573-576.

Cornalba H., Griffiths P. - Analytic cycles and vector bundles in non compact algebraic varieties. Invent. Math. 28 (1975),1-106.

Godement R. - Topologie algébrique et théorie des faisceaux. Activités scientifiques et industrielles (1958), Hermann Paris.

Grauert H. - Ein theorem der analytischen Garben-theorie und die modulräume komplexe Structuren. IHES Sci. Publ. Math. n5 (1960)

Grothendieck A. - SGA 5, théorie des intersections et théorème de Riemann-Roch. Lecture Notes in Math 225, Springer Verlag (1971).

Hirzebruch F. - Neue topologische Methoden in der algebraiche geometrie. Springer Verlag, Berlin.

Hörmander L. - The Analysis of Linear Partial Differential Operators, vol. III et IV, Grundlehren der Math. Wiss. 124.

Houzel Ch., Schapira P. - Images directes de modules différentiels, C.R.A.S.298 (1984), 461-464.

Hurewicz W., Wallman H. - Dimension theory, Ann. of Math. series $n^{\circ} 4$, Princeton University Press (1941).

Jänich K. - Vektorraumbündel und das Raum der Fredholm operatoren. Math. Ann. 161 (1965), 129-142

Kashiwara M. 1.- Index theorem for a maximally overdetermined system of linear differential equations, Proc. Jap. Acad. 49-10 (1973), 803-804.

2.- b-fonctions and holonomic systems. Invent. Math. 38 (1976), 33-54.

3.- Analyse microlocale du noyau de Bergman. Séminaire Goulaouic-Schwartz 1976-77, exp.n8, Ecole Polytechnique.

4.- Introduction to the theory of hyperfunctions. In Seminar on microlocal analysis, Princeton University Press (1979) 3-38.

5.- Systems of microdifferential equations, Progress in Math. 34, Birkhäuser (1983)

Kashiwara M., Kawai T., Kiwura T. - Foundations of algebraic analysis. Princeton Math.

Series $n^{\circ} 37$, Princeton University Press, Princeton N.J. (1986) 
Kashiwara M., Kawai T., Sato M. - Microfunctions and pseudodifferential equations, Lecture Notes 287 (1973), 265-524, Springer-Verlag.

Kashiwara M., Schapira P. 1.- Microlocal study of sheaves. Astérisque 128 (1985).

2.- Sheaves on manifolds, Grundlehren der mathematischen Wissenschaften 292, Springer (1990).

Laumon G. - Sur la catégorie dérivée des D-modules filttés. thèse, Orsay 1983.

Laurent Y. - Théorie de la deuxième microlocalisation dans le domaine complexe, Progress in Math., vol. 53, Birkhäuser, 1985.

Levy R.N. - Riemann-Roch theorems for complex spaces. Acta Math. 158 (1987) 149-188.

Malgrange B. - Sur les images directes de D-modules. Manuscripta Math. 50 (1985),49-71.

Melin A., Sjöstrand J. - Fourier Integral operators with complex valued phase functions. Lecture Notes 459 (1974) 120-223.

Ohana M. - Ellipticité et K-théorie. Note aux C.R.A.S, à paraître.

Pham F. - Singularités des systèmes différentiels de Gauss-Manin. Progress in Math. n², Birkhäuser (1980).

Schapira P. - Microdifferential systems in the complex domain. Grundlehren der mathematischen Wissenschaften 269, Springer (1985).

Schapira P., Schneiders J.P. 1.- Paires elliptiques I - Finitude et dualité C.R. Acad. Sci. 311 (1990) 83-86.

2- Paires elliptiques II -Classes d'Euler et indice, C.R. Acad. Sci. 312 (1991) 81-84.

Segal G. - Fredholm complexes. Quat. J. Math. Oxford Series 21 (1970) 385-402

Steenrod N. - The topology of fibre bundles. Ann. of Math. series $n^{\circ} 144$, Princeton University Press (1951).

Verdier J.L. - Catégories dérivées, état 0. in SGA 4 1/2, Springer Lecture Notes in Math ${ }^{\circ}$ 569 (1977), 262-311. 